\title{
Synchronous-Asynchronous Encounter Probability Analysis of High-Low Runoff for Jinsha River, China, using Copulas
}

\author{
CHEN Jing ${ }^{1,2}$, GU Shixiang ${ }^{1,2 *}$, ZHANG Tianli ${ }^{1,2}$ \\ ${ }^{1}$ Yunnan Water Conservancy and Hydroelectric Survey Design and Research Institute, Kunming 650021, China \\ ${ }^{2}$ Key Laboratory of urban and rural water safety and water saving and emission reduction in Yunnan, Kunming 650201, China
}

\begin{abstract}
Synchronous-asynchronous encounter probability analysis of high-low runoff, which requires a description of the probabilistic properties of hydrological variables, is important in regional water resources management. This study aims to investigate this encounter probability for Jinsha River and its tributary Yalong River in southwest China. A bivariate distribution is used to model the runoff variables of the two rivers based on Copula theory. The Copula is a function that links the univariate marginal distributions to form the bivariate distribution. The bivariate distribution is then employed to determine joint and conditional probabilities. The study results indicate the encounter probability of mainstream runoff and tributary runoff in different periods, also illustrate the mainstream runoff distribution under the condition of knowing the tributary runoff distribution.
\end{abstract}

\section{Introduction}

The Jinsha River, which originates in the Tanggula Mountains in Qinghai province, China, and descends from northwest to southeast through deep valleys of the QTP hinterland, is situated from $24^{\circ} 26^{\prime} \mathrm{N} 90^{\circ} 33^{\prime} \mathrm{E}$ to $36^{\circ} 45^{\prime} \mathrm{N} 105^{\circ} 20^{\prime} \mathrm{E}$ (Fig. 1). The Jinsha River is the largest tributary of the Upper Yangtze River. It has a drainage area of $362 \times 10^{3} \mathrm{~km}^{2}$ with a length of $2290 \mathrm{~km}$, a mean annual runoff discharge of $1410 \times 10^{8} \mathrm{~m}^{3}$, an annual mean precipitation $741 \mathrm{~mm}$ and a mean sediment yield of $2.48 \times 10^{8} \mathrm{t}$. The Jinsha River has many tributaries; most of them are located in the right bank. There are 9 tributaries with the drainage area more than $10 \times 10^{3} \mathrm{~km}^{2}$ and 18 tributaries with the drainage area more than $1.2 \times 10^{3} \mathrm{~km}^{2}$. The Yalong River is the largest tributary of the Jinsha River. The Yalong River, which originates in the Bayanhar Mountains in Qinghai province, is situated from $26^{\circ} 32^{\prime} \mathrm{N} 96^{\circ} 52^{\prime} \mathrm{E}$ to $34^{\circ} 05^{\prime}$ $\mathrm{N} 102^{\circ} 48^{\prime} \mathrm{E}$ and is $1571 \mathrm{~km}$ long with a drainage area of about $129 \times 10^{3} \mathrm{~km}^{2}$ (Fig. 1). It has a mean annual runoff discharge of $570 \times 10^{8} \mathrm{~m}^{3}$ and an annual mean precipitation $726 \mathrm{~mm}$ (see website of the Yangtze River Water Resources Commission of the Ministry of Water Resources, http://www.cjw.gov.cn).

It has been reported the variation phases and its characteristics of hydrological regime of Jinsha River basin. Chen Yuan et al. (2010) analyzed the distribution and annual variation and the tendency, mutation and periodicity of runoff by the stochastic hydrology method, based on the data of runoff in Jinsha River and its tributary Yalong River. The result showed that the Jinsha River had abundant water with distribute unevenly during year, annual variation small and continued high runoff season and low runoff season obviously. Xu Changjiang et al. (2011) illustrated the inter-annual runoff in the basin changes little and the change in the upstream is slightly larger than that in the middle stream and downstream; the annual runoff distribution is uneven and the distribution in upstream is more uneven than that in the downstream; since $1950 \mathrm{~s}$, at confidence level of 0.5 , the check values of the annual runoff series at the main hydrological stations on the main stem have not break the critical values, demonstrating the annual runoff series have no obvious increase and decrease tendency. Zhao Wenhuan and Gao Yuan (2011) analyzed the annual and decadal runoff variation characteristics of Jinsha River basin. The results reveal that from 1940s, the annual and decadal runoff variation remains stable generally; and from 1980s, the runoff in middle reach of Jinsha River shows a slight increasing trend.

Studying runoff variation is the assurance for water resources integrated development, scientific management and optimal operation. Thus, it shows important practical significance on utilizing and maintaining balance of regional water resources by analyzing synchronous-asynchronous encounter probability of high-low runoff. Since the encounter of high-low runoff is a complex phenomenon, the

*Corresponding author, email: gushxang@qq.com. 
bivariate hydrological frequency analysis method is needed. This study focuses on analyzing the encounter probability of Jinsha River and its tributary Yalong River by employing Copulas.

The research history of Copulas can be traced back to 1959. With the developing of computer technology, the theory of Copulas has made rapid progress since the late 1990s. The joint distribution function of multivariate vectors can be written in terms of marginal distribution functions and a Copula function. The Copula function describes the dependence structure between the components and can be used to describe the dependence between random variables.

In the field of hydrology, Michele and Salvadori (2003) indicated how the use of bivariate Copulas can be used to reproduce both the marginal variability of storm average intensity and duration. Michele et al. (2004) applied bivariate Copulas concept to flood peak and volume positively correlated for checking the adequacy of dam spillway. Favre et al. (2004) applied Copulas to bivariate frequency analysis into two different problems (i.e., flow combination and joint modeling of flow and volume) in hydrology. Zhang and Vijay (2007) analyzed the bivariate rainfall frequency distributions to ensure joint and conditional return periods. Two doctoral theses (Zhang, 2005; Xiao, 2007) have made a detailed exposition on Copulas and based on this method undertaking the hydrological frequency analysis of flood, precipitation and drought. Guo, et al. (2008) explored the applicability and superiority in multivariate hydrological analysis and estimation of Copulas.

Copula theory is a realistic way to handle the problem of bivariate variables encounter probability analysis. In this paper, the purpose is achieved through using Copulas to construct bivariate runoff distributions. Based on this joint probability distribution, the runoff encounter probability of Jinsha River and its tributary Yalong River is investigated and the conditional probability distributions are also explored.

The next section illustrates the observation data of study area. Section 3 is devoted to the general theory about Copulas. The definition and basic theorem of Copulas have been introduced, and the main types of Copulas, the Archimedean Copulas, have been presented. Section 4 deals with the encounter probability analysis model construction based on Archimedean Copulas. Section 5 discusses the study results of synchronous-asynchronous encounter probability analysis of high-low runoff. Section 6 is devoted to the conclusions. 


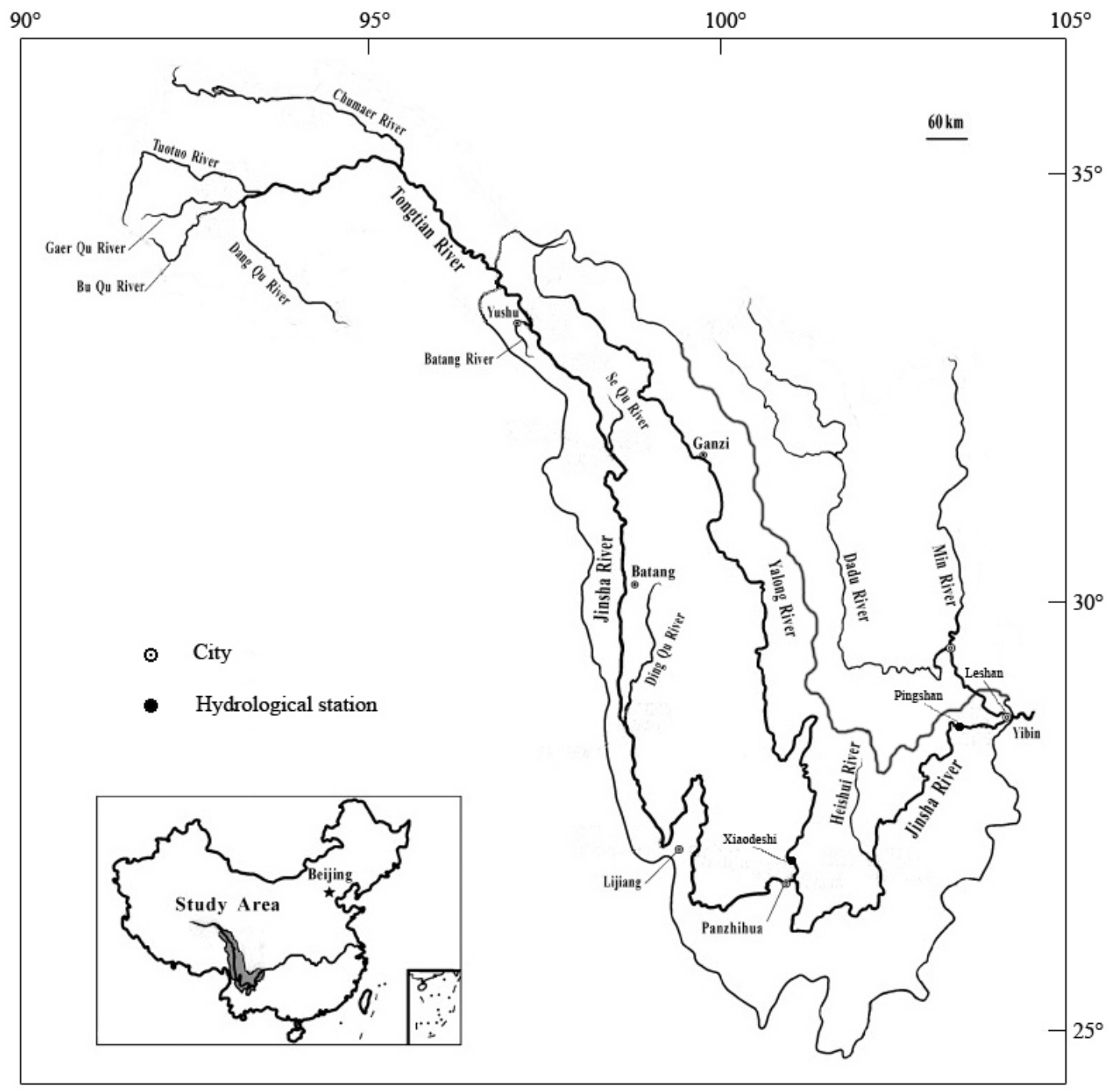

Fig. 1 Map of the Jinsha River basins and the control hydrological stations locations

\section{Data used}

The data used in this study to evaluate synchronousasynchronous encounter probability of high-low runoff are the monthly runoff data (from 1953 to 2004) of the Pingshan hydrological station in Jinsha River and the
Xiaodeshi hydrological station in Yalong River (see Fig. 1).

We divided the data into three periods: the flood period (by the impact of changes in seasonal precipitation, the flood period of the Jinsha River and the Yalong River is June to October), non-flood period (November to next May) and the whole year. The discharge hydrograph are given in Fig. 2.



(a)

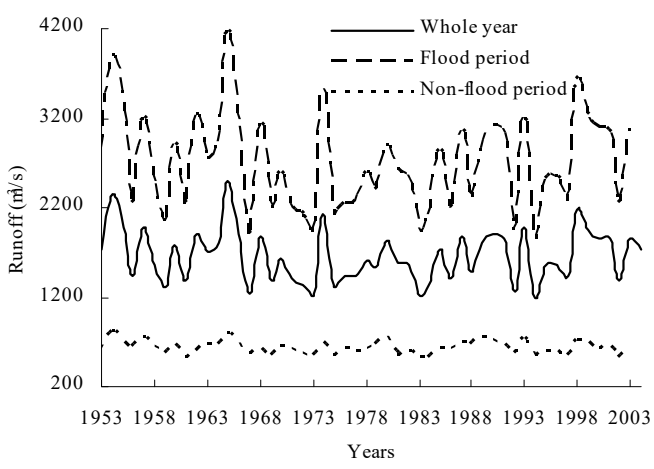

(b)

Fig. 2 The discharge hydrograph of Pingsha and Xiaodeshi station: (a) the runoff of Pingshan station and (b) the runoff of Xiaodeshi station. 
The classification of high-low runoff is based on the amount of monthly runoff in different periods. It is important for the analysis of synchronousasynchronous encounter probability. According to the China national standards: Standard of essential technical terms and symbols in hydrology (GBPT50095-98), the definition is given as: (a) the high runoff, means that the amount of runoff is significantly greater than the statistical average, (b) the normal runoff, means that the amount of runoff is approximately equal to the statistical average, and (c) the low runoff, means that the amount of runoff is significantly less than the statistical average.

In terms of statistic frequency analysis, the high runoff is defined as $Q_{i}>Q_{p}=37.5 \%$ and the low runoff is defined as $Q_{i}<Q_{p=62.5 \%}$, in which, $Q_{i}$ is the annual runoff for $i$-year. $Q_{p=37.5 \%}$ and $Q_{p=62.5 \%}$ stand for the annual runoff of scenario $p=37.5 \%$ and scenario $p=62.5 \%$, respectively, which $p$ is the empirical frequency for annual runoff sequences (Yang, 2000).

\section{General Copula theory}

Construction of multivariate joint distribution is reduced to probe the relationship among the correlated random variables when marginal probability distributions are given. This is very difficult. Copulas, developed by Sklar (1959), are functions that connect multivariate probability distributions to their onedimensional marginal probability distributions (Nelsen, 1999). The advantage of using Copulas to construct multivariate distributions is that Copulas can separate the effects of dependence from the effects of marginal and it also gives great freedom in choosing the univariate marginal distributions. The further merit of using Copulas is that it is easily extended to an $\mathrm{N}$ dimensional case, i.e. $N$-variate distribution, although this is not a topic considered in this study.
Consider bivariate random variables $X$ and $Y$ with marginal distribution functions as $F_{X}(x)=P(X \leq x)$ and $F_{Y}(y)=P(Y \leq y)$, where $x$ and $y$ are the value of $X$ and $Y$. Thus, the joint distribution (the bivariate probability distribution), $H$, is expressed in terms of its marginal functions and the Copula function. That is, the Copula function couple multivariate distribution functions to their one-dimensional marginal distribution functions. Sklar (1959) described this most important property which is now known as Sklar's theorem (twodimensional):

$$
P(X \leq x, Y \leq y)=H(x, y)=C(u, v)=C\left(F_{X}(x), F_{Y}(y)\right)(1)
$$

Conversely, for any univariate distributions $F_{X}(x)$ and $F_{Y}(y)$ and any Copula function $C$, the function $H$ defined above is a two-dimensional distribution function with marginal distributions $F_{X}(x)$ and $F_{Y}(y)$. Furthermore, if $F_{X}(x)$ and $F_{Y}(y)$ are continuous, then $C$ is unique.

There are a multitude of Copula functions. Generally speaking, the most popular class of Copula functions, applied in several disciplines, is the Archimedean Copulas. Archimedean Copulas are desirable for hydrologic analysis for the following properties (the proofs have been reported by Genest and Mackay 1986): (a) they can be easily constructed; (b) a large varieties of Copula functions belong to this family; and (c) Archimedean Copula can be applied when the correlation amongst hydrologic variables is positive or negative. For this reason the one-parameter Archimedean Copula were applied in this study.

Let $\varphi(\cdot)$ be a continuous, strictly decreasing function from $[0,1]$ to $[0, \infty)$ such that $\varphi(1)=0$ and let $\varphi^{[-}$ ${ }^{1]}(\cdot)$ be the pseudo-inverse of $\varphi(\cdot)$. Then $C$ is the Archimedean Copula function which given as

$C(u, v)=\varphi^{[-1]}(\varphi(u)+\varphi(v))$

where $\varphi(\cdot)$ is called generator and $\forall u, v \in[0,1]$. There are three commonly used one-parameter Archimedean Copulas are given in Table 1.

Table 1 Three one-parameter Archimedean Copulas

\begin{tabular}{|c|c|c|c|c|}
\hline Type & Generator & Function & Parameter range & $\begin{array}{l}\text { Relationship between } \\
\tau \text { and the parameter }\end{array}$ \\
\hline Gumbel & $(-\ln u)^{1 / \alpha}$ & $C_{G}(u, v)=\exp \left(-\left[(-\ln u)^{\frac{1}{\alpha}}+(-\ln v)^{\frac{1}{\alpha}}\right]^{\alpha}\right)$ & $\alpha \in(0,1]$ & $\tau=1-\alpha$ \\
\hline Clayton & $u^{-\theta}-1$ & $C_{C l}(u, v)=\left(u^{-\theta}+v^{-\theta}-1\right)^{-1 / \theta}$ & $\theta \in(0, \infty)$ & $\tau=\theta /(\theta+2)$ \\
\hline Frank & $-\ln \frac{e^{-\lambda u}-1}{e^{-\lambda}-1}$ & $C_{F}(u, v)=-\frac{1}{\lambda} \ln \left(1+\frac{\left(e^{-\lambda u}-1\right)\left(e^{-\lambda v}-1\right)}{e^{-\lambda}-1}\right)$ & $\lambda \neq 0$ & $\tau=1+\frac{4}{\lambda}\left(\frac{1}{\lambda} \int_{0}^{\lambda} \frac{t}{e^{t}-1} d t-1\right)$ \\
\hline
\end{tabular}

In Table 1, $\tau$ is Kendall's coefficient of correlation between $X$ and $Y$, which is determined from observations:

$$
\tau=\left(\begin{array}{c}
m \\
2
\end{array}\right)^{-1} \sum_{i<j} \operatorname{sign}\left[\left(x_{i}-x_{j}\right)\left(y_{i}-y_{j}\right)\right] \quad(i, j=1,2, \cdots, m)
$$

where $m$ is the number of observations; sign $=1$ if $\left(x_{i^{-}}\right.$ $\left.x_{j}\right)\left(y_{i^{-}} y_{j}\right)>0$, sign $=-1$ if $\left(x_{i^{-}} x_{j}\right)\left(y_{i^{-}} y_{j}\right)<0$ and $\operatorname{sign}=0$ if $\left(x_{i^{-}}\right.$ $\left.x_{j}\right)\left(y_{i}-y_{j}\right)=0$.

To identify an appropriate Archimedean Copula, two tests based on the empirical Copula have been presented by Christian Genest (2007). Firstly the empirical Copula function $C_{E m p}$ is defined to directly reflect the spatial distribution characteristics of the given observations, which is given as:

$C_{E m p}=$ number of $\left(x_{1 j}, x_{2 j}\right)$ such that $x_{1 j}<x_{1 i}$ and $\left.x_{2 j}<x_{2 i}\right\} /(m+1) \quad(i=1,2, \ldots, m)$. (4) 
Then the goodness-of-fit test (such as ordinary least squares standard, OLS standard) and KolmogorovSmirnov test (K-S test) are used to make sure weather the identified Copula is adequate for the empirical Copula. Otherwise, the Copula function needs to be reidentified.

By calculating the sum of squared vertical distances between the values of $C_{E m p}$ and $C$, the OLS standard considers the Copula which has the minimum calculation as the identified Copula (Wang et al., 2009).

The K-S test is applied to determine if a random variable could have the hypothesized, continuous, cumulative distribution function; it uses the maximum absolute difference between the empirical distribution and the hypothesized probability distribution (Yevjevich, 1972), that is, the K-S test is used to determine whether the values after applying the identified Copula could be fit for the empirical Copula.

\section{Encounter probability analysis model based on Archimedean Copula}

By building the joint distribution based on Archimedean Copula, the correlation of variables can be studied. Application and testing of the Archimedean Copulas in synchronous-asynchronous encounter probability analysis of high-low runoff involve: (a) determination of marginal distributions based on the conventional statistical approach in hydrology, (b) identification of parameter and generator of Archimedean Copulas, (c) determination of the joint probability distribution, and (d) application to real data.

The advantage of Copulas is that no assumption is needed for the hydrological variables to be independent or normal or have the same type of marginal distributions. According to the conventional statistical approach, we can choose the appropriate mathematical statistical model to describe the marginal distributions of runoff variables in Jinsha River and Yalong River. The Pearson Type III distribution is suited to analyze the frequency calculations of hydrologic variables in China (Ye and Zhan, 2003; Jin, 2010). The Pearson Type III distribution is a reparameterized form of the three-parameter gamma distribution, and can be fit to a wide range of shapes with positive or negative skewness, including a good approximation to the normal distribution.

For the random variable $X$ (likewise the random $Y$ ), the cumulative distribution function of Pearson Type III is given as:

$$
\left.P=P(X \geq x)=1-F(x)=\frac{\beta^{\alpha_{X}}}{\Gamma\left(\alpha_{X}\right)} \int_{x}^{\infty}\left(x-a_{0, X}\right)^{\alpha_{X}-1} \exp -\beta_{X}\left(x-a_{0, X}\right)\right) d x
$$

where $\Gamma$ denotes the gamma function. $n$ means the mark of the variable which is distinguished from each other. $\alpha_{X}=4 / C s_{X}{ }^{2} \quad, \quad \beta_{X}=2 /\left(\bar{x} \times C v_{X} \times C s_{X}\right) \quad$ and $a_{0, X}=\bar{x}\left(1-2 C v_{X} / C s_{X}\right)$ are shape, scale and location parameters. For a random observation sample of the variable $X, \bar{x}$ is the mean, $C v_{X}$ is the coefficient of variation and $C s_{X}$ is the skewness coefficient.

In this paper, the probability weighted moment (PWM) method (Ding et al., 1989) was applied to the Pearson Type III distribution for estimating parameters $\bar{x}, C v_{X}$ and $C s_{X}$ (likewise the parameters $\bar{y}, C v_{Y}$ and $\left.C s_{Y}\right)$. With objective principle and simple calculation, The PWM method adopts low-power computing for reducing error, and can make good use of the sample information.

Let $X$ be the runoff of Pingshan hydrological station in Jinsha River and $Y$ be the runoff of Xiaodeshi hydrological station in Yalong River. Consider observation data $\left\{x_{i}\right\}_{i=1}^{m}$ and $\left\{y_{i}\right\}_{i=1}^{m} \quad(m=52)$ of variables $X$ and $Y$. The marginal distribution functions $F_{X}(x)$ and $F_{Y}(y)$ accord with the cumulative distribution function of Pearson Type III. Then the synchronousasynchronous encounter probability analysis of highlow runoff model based on Archimedean Copula can be given as:

$$
\begin{aligned}
& x_{i}=\bar{x}\left(1+C v_{X} \Phi_{X, i}\right) \quad(6 \mathrm{a}) \\
& y_{i}=\bar{y}\left(1+C v_{X} \Phi_{X, i}\right) \quad(6 \mathrm{~b}) \\
& \left(x_{i}, y_{i}\right) \sim C\left(F_{X}\left(x_{i}\right), F_{Y}\left(y_{i}\right)\right) \quad i=1,2, \cdots, m
\end{aligned}
$$

where $\Phi$ is called coefficient of deviation from the mean, with $\Phi_{X}=\left(x_{i}-\bar{x}\right) / \bar{x} / C v_{X}$ and

$$
\Phi_{Y}=\left(y_{i}-\bar{y}\right) / \bar{y} / C v_{Y} \text {. }
$$

\section{Results and discussion}

\section{1 model foundation}

In this section, the aim is to estimate the joint probability distribution of $X$ and $Y$ taking into analysis the synchronous-asynchronous encounter probability of high-low runoff. The Archimedean Copula is applied to model the monthly runoff data from 1953 to 2004.

The first step of the procedure is to identify the marginal distributions of the two variables. The Pearson Type III distribution (see formula 5) is adopted and its parameters are estimated by the PWM method. The estimation results are listed in Table 2. Empiric frequencies, calculated by expectation formula $P=i$ $/(m+1)(i=1,2, \ldots, m)$, have been fitted with the Pearson Type III distribution (see Fig. 3). Good data fitting results in Fig. 3 illustrates that it is acceptable to description the marginal distributions by using the Pearson Type III. 
Table 2 Parameters of the Pearson Type III distribution

\begin{tabular}{|c|c|c|c|c|c|c|}
\hline \multirow{2}{*}{ Period } & \multicolumn{3}{|c|}{ Pingshan station of Jinsha River } & \multicolumn{3}{|c|}{ Xiaodeshi station of Yalong River } \\
\hline & $\bar{x}$ & $C v_{X}$ & $C s_{X}$ & $\bar{y}$ & $C v_{Y}$ & $C s_{Y}$ \\
\hline Flood period & 7183.79 & 0.185 & 0.504 & 2684.66 & 0.203 & 0.488 \\
\hline Non-flood period & 1940.36 & 0.134 & 0.540 & 634.48 & 0.116 & 0.377 \\
\hline Whole year & 4562.08 & 0.168 & 0.457 & 1659.57 & 0.183 & 0.482 \\
\hline
\end{tabular}
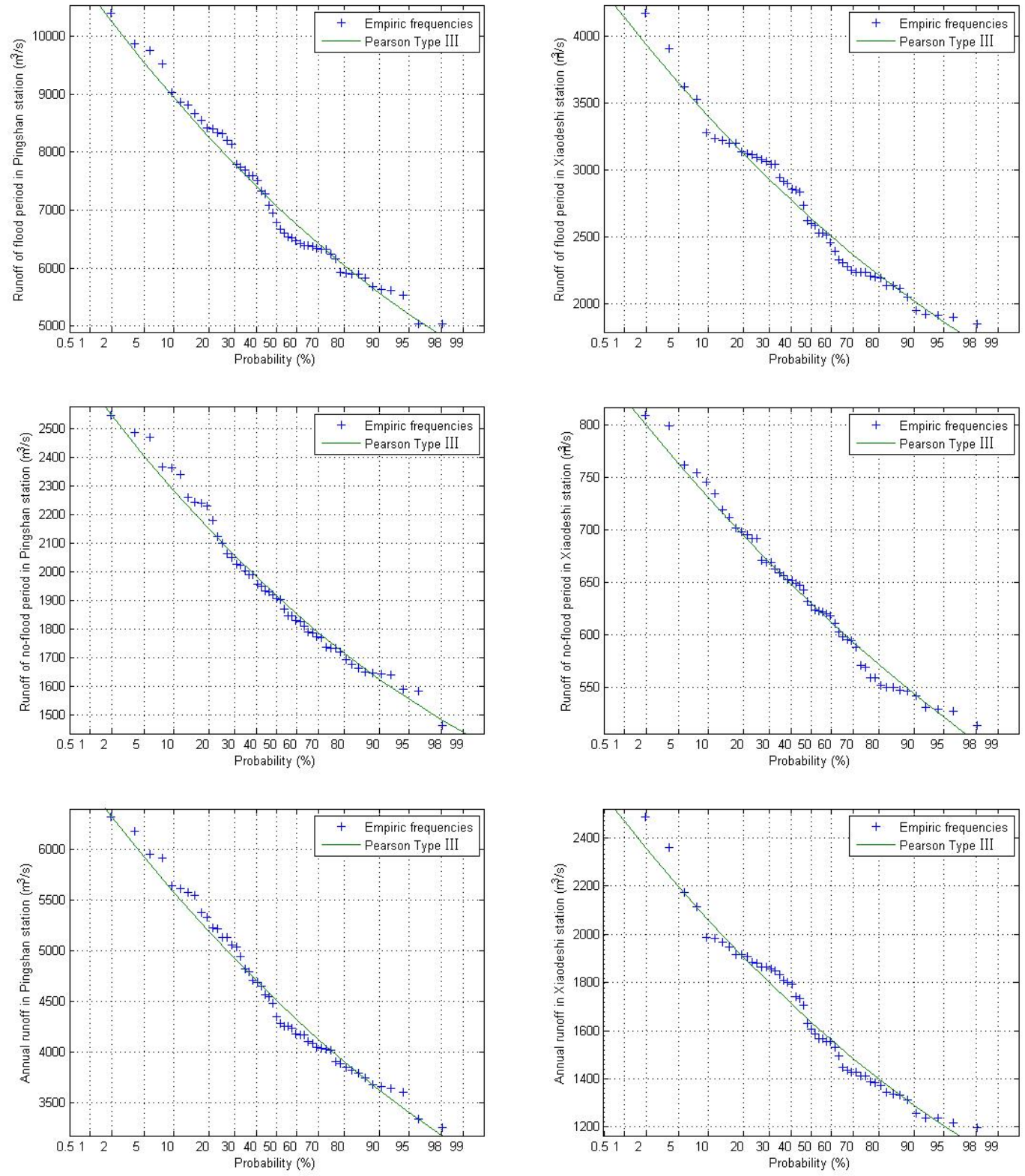

Fig. 3 Marginal distributions curve fitting

The second step is to characterize the correlation structure of the available sample. Kendall's $\tau$ values estimated on the $X$ and $Y$ observed variables in the flood period, non-flood period and the whole year are respectively equal to $0.7498,0.5639$ and 0.7333 . Then, there is correlation among all quantities, denoting asymmetric bivariate structures of dependence.

The third step is to estimate the $\alpha, \theta$ and $\lambda$ parameters of three one-parameter Archimedean Copulas according to the relationship with Kendall's $\tau$. Table 3 shows the calculation results.
The forth step is the choice of the best fitting Archimedean Copula among those estimated. At first, defining the empirical Copula function $C_{E m p}$ based on formula (4). Then using the OLS standard and the K-S test identify the appropriate Copula. The results are listed in Table 3 . The $\mathrm{K}-\mathrm{S}$ statistic and $P$-value indicate that the three adopted Archimedean Copulas in each period can pass the K-S test and are available for describing the joint probability distribution of bivariate random variables $X_{1}$ and $X_{2}$. According to the minimum OLS calculations (the bold values in Table 3), in the 
non-flood period, the Clayton Copula is identified; in the other two periods, the Frank Copula is identified.

Table 3 shows that the OLS value in the non-flood period based on the Frank Copula is approximately equal to the one based on the Clayton Copula ( 0.0012 and 0.0010 ). In order to maintain consistency, the Frank Copula is finally identified as the adopted Copula for making the synchronous-asynchronous encounter probability analysis in three periods in this paper.

Plots distributions of Frank Copula function $C_{F}$ and empirical Copula function $C_{E m p}$ for the given observation sample are compared in Fig. 4, which shows a good fitting effect.

Table 3 The parameters estimation and the best fitting identification of three Archimedean Copulas

\begin{tabular}{|c|c|c|c|c|}
\hline \multirow{3}{*}{ Period } & \multicolumn{4}{|c|}{ Gumbel Copula } \\
\hline & \multirow{2}{*}{$\alpha$} & \multicolumn{2}{|c|}{ K-S test } & \multirow{2}{*}{ OLS } \\
\hline & & K-S statistic & $P$-value & \\
\hline Flood period & 0.2502 & 0.1823 & 0.6844 & 0.0032 \\
\hline Non-flood period & 0.4361 & 0.0874 & 0.8132 & 0.0016 \\
\hline Whole year & 0.2667 & 0.1848 & 0.6542 & 0.0131 \\
\hline \multirow{3}{*}{ Period } & \multicolumn{4}{|c|}{ Clayton Copula } \\
\hline & \multicolumn{3}{|c|}{$\mathrm{K}$-S test } & \multirow{2}{*}{ OLS } \\
\hline & 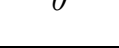 & K-S statistic & $P$-value & \\
\hline Flood period & 5.9936 & 0.1761 & 0.6910 & 0.0026 \\
\hline Non-flood period & 2.5861 & 0.0830 & 0.8595 & 0.0010 \\
\hline Whole year & 5.4990 & 0.1734 & 0.72154 & 0.0121 \\
\hline \multirow{3}{*}{ Period } & \multicolumn{4}{|c|}{ Frank Copula } \\
\hline & \multicolumn{3}{|c|}{ K-S test } & \multirow{2}{*}{ OLS } \\
\hline & $n$ & K-S statistic & $P$-value & \\
\hline Flood period & 30.8775 & 0.1062 & 0.7734 & 0.0023 \\
\hline Non-flood period & 5.8278 & 0.0862 & 0.8263 & 0.0012 \\
\hline Whole year & 31.1277 & 0.0977 & 0.8068 & 0.0106 \\
\hline
\end{tabular}

Note: $P$-value is the probability of the maximum K-S statistic being greater than or equal to the critical; the critical value for the test is 0.05 .

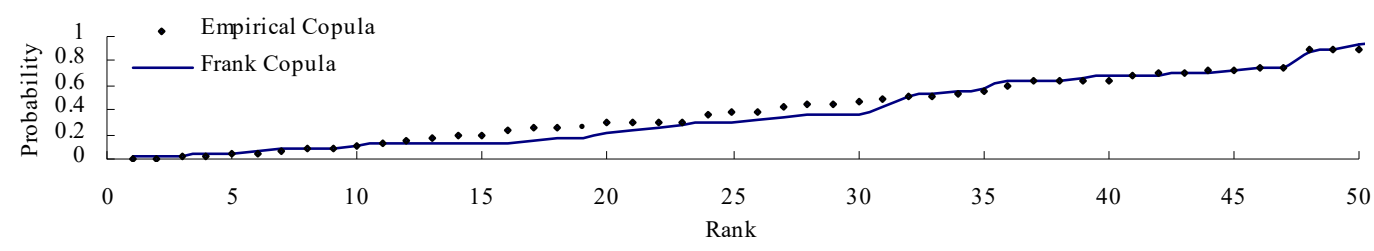

(a)



(b)

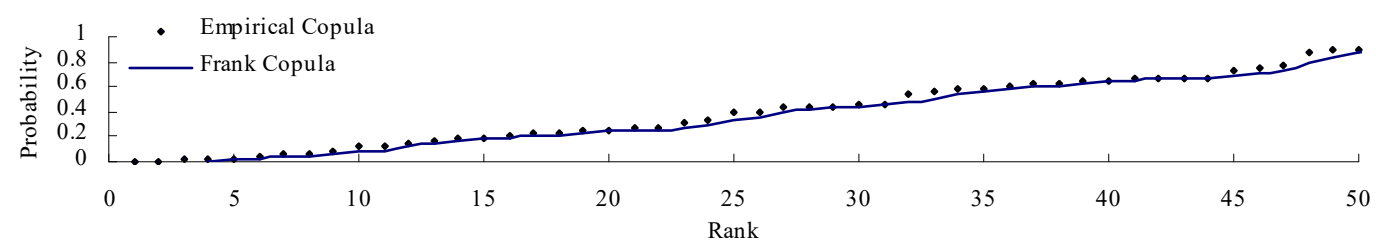

(c)

Fig. 4 Comparison of Frank Copula function $C_{F}$ and empirical Copula function $C_{E m p}$ : (a) the flood period; (b) the no-flood period; and (c) the whole year. 


\subsection{Encounter probability analysis}

The joint probability distribution of bivariate runoff variables are shown in Fig. 5 which indicate the encounter probability of mainstream runoff and

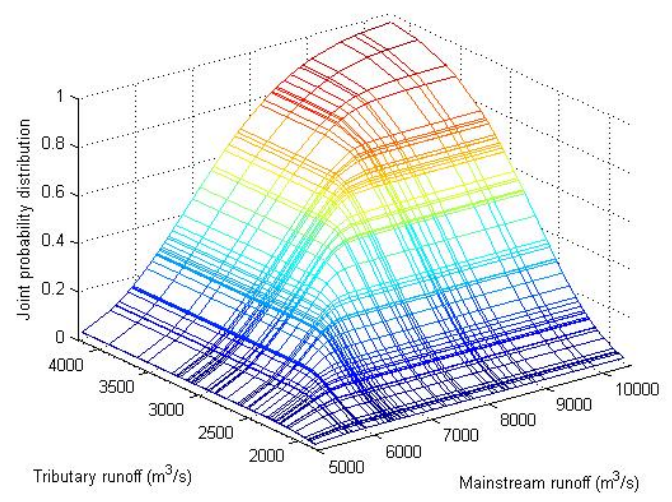

(a)

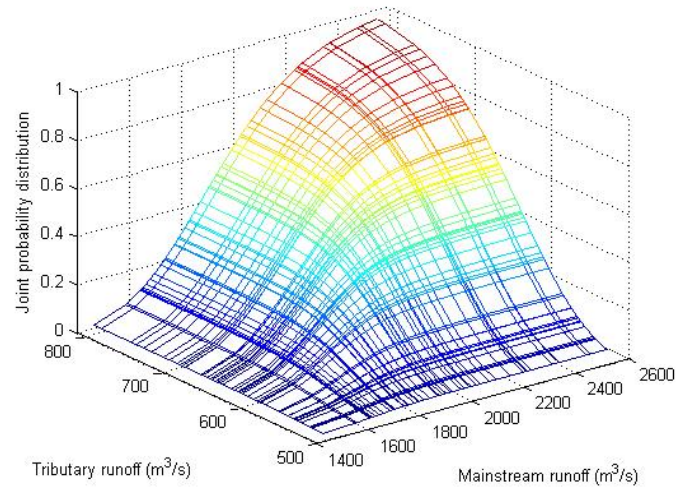

(b)



(c)

Fig. 5 Joint probability distributions: (a) the flood period; (b) the no-flood period; and (c) the whole year.

The synchronous-asynchronous encounter probability of high-low runoff includes 9 types: (a) $p_{1}$, probability of mainstream high runoff encounters tributary high runoff; (b) $p_{2}$, probability of mainstream high runoff encounters tributary normal runoff; (c) $p_{3}$, probability of mainstream high runoff encounters tributary low runoff; (d) $p_{4}$, probability of mainstream normal runoff encounters tributary high runoff; (e) $p_{5}$, probability of mainstream normal runoff encounters
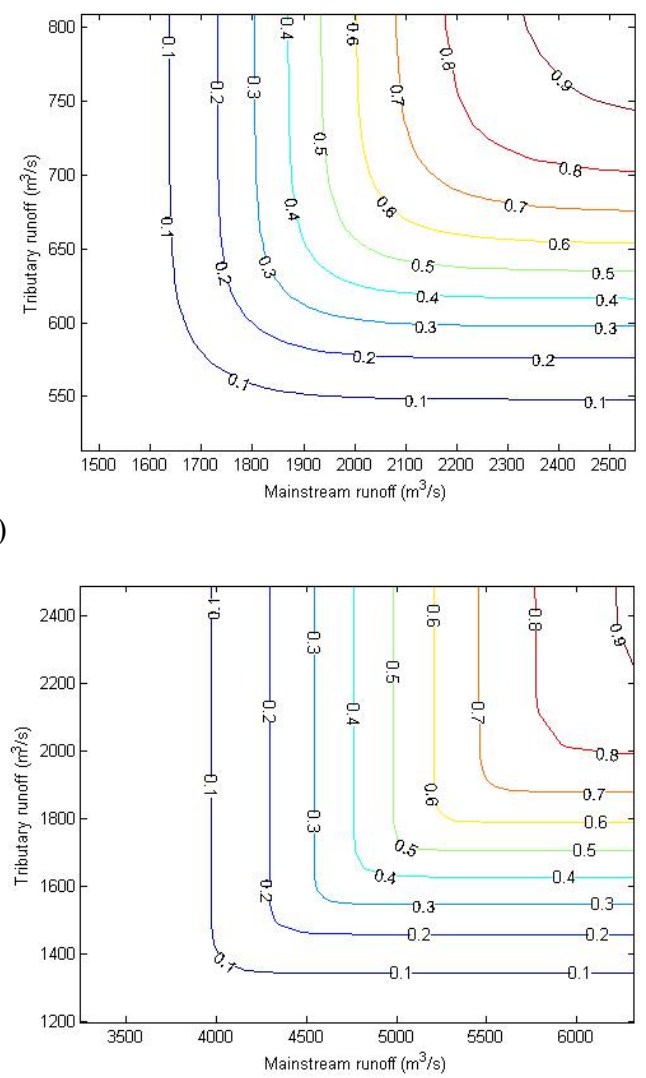

tributary runoff in different periods. According to the isogram of joint distribution, it is illustrated the probabilities of various encounter events. For instance, when the mainstream runoff is less than or equal to $2000 \mathrm{~m}^{3} / \mathrm{s}$ and the tributary runoff is less than or equal to $700 \mathrm{~m}^{3} / \mathrm{s}$ in no-flood period, the encounter probability is $59.18 \%$.

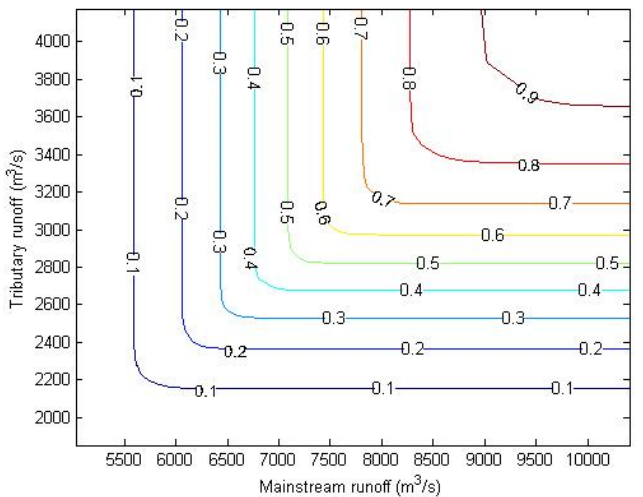

tributary normal runoff; (f) $p_{6}$, probability of mainstream normal runoff encounters tributary low runoff; (g) $p_{7}$, probability of mainstream low runoff encounters tributary high runoff; (h) $p_{8}$, probability of mainstream low runoff encounters tributary normal runoff; and (i) $p_{9}$, probability of mainstream low runoff encounters tributary low runoff. They can be expressed as: 


$$
\begin{gathered}
p_{1}=P\left(X_{1} \geq x_{1, p h}, X_{2} \geq x_{2, p h}\right)=1-F_{X_{1}}\left(x_{1, p h}\right)-F_{X_{2}}\left(x_{2, p h}\right)+H\left(x_{1, p h}, x_{2, p h}\right) \\
p_{2}=P\left(X_{1} \geq x_{1, p h}, x_{2, p l}<X_{2}<x_{2, p h}\right)=F_{X_{2}}\left(x_{2, p h}\right)-F_{X_{2}}\left(x_{2, p l}\right)-H\left(x_{1, p h}, x_{2, p h}\right)+H\left(x_{1, p h}, x_{2, p l}\right) \\
p_{3}=P\left(X_{1} \geq x_{1, p h}, X_{2} \leq x_{2, p l}\right)=F_{X_{2}}\left(x_{2, p l}\right)-H\left(x_{1, p h}, x_{2, p l}\right) \\
p_{4}=P\left(x_{1, p l}<X_{1}<x_{1, p h}, X_{2} \geq x_{2, p h}\right)=F_{X_{1}}\left(x_{1, p h}\right)-F_{X_{1}}\left(x_{1, p l}\right)-H\left(x_{1, p h}, x_{2, p h}\right)+H\left(x_{1, p l}, x_{2, p h}\right) \\
p_{5}=P\left(x_{1, p l}<X_{1}<x_{1, p h}, x_{2, p l}<X_{2}<x_{2, p h}\right)=H\left(x_{1, p h}, x_{2, p h}\right)-H\left(x_{1, p h}, x_{2, p l}\right)-H\left(x_{1, p l}, x_{2, p h}\right)+H\left(x_{1, p l}, x_{2, p l}\right) \\
p_{6}=P\left(x_{1, p l}<X_{1}<x_{1, p h}, X_{2} \leq x_{2, p l}\right)=H\left(x_{1, p h}, x_{2, p l}\right)-H\left(x_{1, p l}, x_{2, p l}\right) \\
p_{7}=P\left(X_{1} \leq x_{1, p l}, X_{2} \geq x_{2, p h}\right)=F_{X_{1}}\left(x_{1, p l}\right)-H\left(x_{1, p l}, x_{2, p h}\right) \\
p_{8}=P\left(X_{1} \leq x_{1, p l}, x_{2, p l}<X_{2}<x_{2, p h}\right)=H\left(x_{1, p l}, x_{2, p h}\right)-H\left(x_{1, p l}, x_{2, p l}\right) \\
p_{9}=P\left(X_{1} \leq x_{1, p l}, X_{2} \leq x_{2, p l}\right)=H\left(x_{1, p l}, x_{2, p l}\right)
\end{gathered}
$$

According to the aforementioned, frequencis for types are classified into two kinds of cases: the defining the high runoff, the normal runoff and the low runoff are given as: $p h=62.5 \%$ and $p l=37.5 \%$. The 9

synchronous and the asynchronous encounter probability. The calculation results are listed in Table 4.

Table 4 The synchronous-asynchronous encounter probability of high-low runoff

\begin{tabular}{cccccccccccc}
\hline \multirow{2}{*}{ Period } & \multicolumn{3}{c}{ Synchronous encounter probability (\%) } & \multicolumn{6}{c}{ Asynchronous encounter probability (\%) } \\
\cline { 2 - 12 } & $p_{1}$ & $p_{5}$ & $p_{9}$ & Sum & $p_{2}$ & $p_{3}$ & $p_{4}$ & $p_{6}$ & $p_{7}$ & $p_{8}$ & Sum \\
\hline Flood period & 34.58 & 13.45 & 28.38 & 76.42 & 0.07 & 0 & 12.32 & 0.11 & 0.05 & 11.03 & 23.58 \\
No-flood period & 26.45 & 9.88 & 26.41 & 62.74 & 7.51 & 2.58 & 8.34 & 7.55 & 2.98 & 8.30 & 37.26 \\
Whole year & 43.28 & 10.76 & 20.31 & 74.35 & 14.32 & 0.11 & 0.04 & 11.08 & 0 & 0.10 & 25.65 \\
\hline
\end{tabular}

Table 4 illustrates the following characteristics of Jinsha River (mainstream) and Yalong River (tributary) runoff encounter probability: (a) in the three periods, the synchronous encounter probability is significantly higher than the asynchronous encounter probability; (b) compared with the synchronous low runoff encounter probability, the synchronous high runoff encounter probability is higher, and the synchronous normal runoff encounter probability is lower; (c) in the flood period, they are high probability events that mainstream normal runoff encounters tributary high runoff and mainstream low runoff encounters tributary normal runoff, but it is almost zero probability event that mainstream high runoff encounters tributary low runoff; (d) in the no-flood period, the events that mainstream high runoff encounters tributary normal runoff, mainstream normal runoff encounters tributary high runoff, mainstream normal runoff encounters tributary low runoff and mainstream low runoff encounters tributary normal runoff frequently happen; and (e) in the whole year, the events that mainstream high runoff encounters tributary normal runoff and mainstream normal runoff encounters tributary low runoff are most likely to occur, and the events that mainstream low runoff encounter tributary high runoff is most unlikely to occur.

Finally, it is necessary to analyze mainstream runoff distribution under the condition of knowing the tributary runoff distribution, that is, to analyze the conditional probability. When $X_{2} \geq x_{2, p h}, x_{2, p l}<X_{2}<x_{2, p h}$ and $X_{2} \leq x_{2, p l}$, the conditional probability of $X_{1}$ can be respectively given as:

$$
\begin{gathered}
P_{1, X_{1} \mid X_{2}}\left(x_{1}, x_{2}\right)=P\left(X_{1} \geq x_{1} \mid X_{2} \geq x_{2, p h}\right)=1-\frac{F_{X_{1}}\left(x_{1}\right)-H\left(x_{1}, x_{2, p h}\right)}{1-F_{X_{2}}\left(x_{2, p h}\right)} \\
F_{2, X_{1} \mid X_{2}}\left(x_{1}, x_{2}\right)=P\left(X_{1} \geq x_{2} \mid x_{2, p l}<X_{2}<x_{2, p h}\right)=1-\frac{H\left(x, x_{2, p h}\right)-H\left(x, x_{2, p l}\right)}{F_{X_{2}}\left(x_{2, p h}\right)-F_{X_{2}}\left(x_{2, p l}\right)} \\
F_{3, X_{1} \mid X_{2}}\left(x_{1}, x_{2}\right)=P\left(X_{1} \geq x_{1} \mid X_{2} \leq x_{2, p l}\right)=1-\frac{H\left(x, x_{2, p l}\right)}{F_{X_{2}}\left(x_{2, p l}\right)}
\end{gathered}
$$

Fig. 6 shows these three conditional probability distributions in different periods. It illustrate that the probabilities of various mainstream runoff in three periods can be ensured under the giving conditions, in which, the tributary runoff is high, normal or low. For instance, when the tributary runoff is high in flood period, the probability that mainstream runoff is greater than or equal to $8000 \mathrm{~m}^{3} / \mathrm{s}$ is $74.21 \%$. 

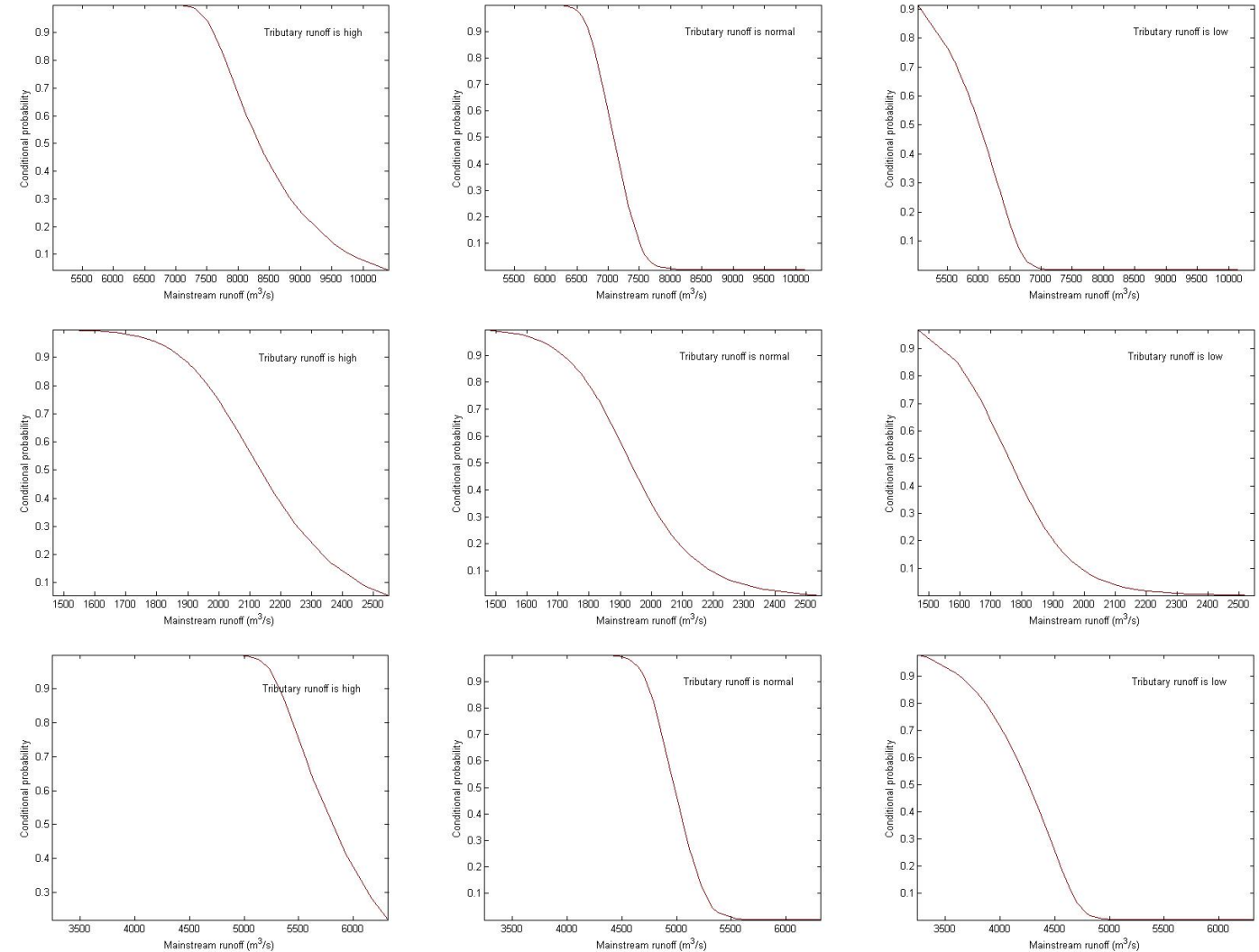

Fig. 6 Conditional probability distributions: (a) the flood period; (b) the no-flood period; and (c) the whole year.

\section{Conclusions}

An analysis of synchronous-asynchronous encounter probability of high-low runoff for Jinsha River and its tributary Yalong River in southwest China is provided. On the basis of Copulas, monthly runoff data of two hydrological stations which are located in the outlet of the two rivers is used to construct the bivariate distribution from the univariate marginal distributions of runoff variables.

The Copulas play an important role in multivariate analyses nowadays. The Copula function is a mapping uniquely determined whenever the marginal distribution functions are continuous, and captures the essential features of the dependence between random variables. Application and testing of the method involve: (a) determination of marginal distributions based on the Pearson Type III distribution; (b) calculation of Kendall's $\tau$ values and identification of parameter of Archimedean Copula, the OLS standard and the K-S test has been adopted; (c) determination of the best fitting Archimedean Copula, and the Frank Copula is finally identified; and (d) application to the given observation sample, an approach based on Frank Copula applied to the encounter probability of Jinsha River and Yalong River analysis is proposed.

The application results show the characteristics of Jinsha River (mainstream) and Yalong River (tributary) runoff encounter probability, which is valuable for the rational allocation and joint operation of regional water resources. In addition, conditional probability distribution which is the mainstream runoff distribution under the condition of knowing tributary runoff distribution has been analyzed. It reveals the variation of mainstream runoff when it is affected by the tributary runoff.

\section{Acknowledgments}

The work was financially supported by the Major projects of national water pollution control and control technology (No. 2013ZX07102-006-01), Yunnan key fund for applied basic research (No.2017FA022), Academician workstation construction special (No.2015IC013), and Yunnan provincial innovation team construction project (No.YKRF2017-07-26).

\section{References}

1. Chen Yuan, Wang Shunjiu, Wang Guoqing, et al. Runoff Variation Characteristics Analysis on Jinsha River [J]. Plateau and Mountain Meteorology Research, 2010, 30(2): 26- 30 .

2. Christian Genest, Bruno Remillard, David Beaudoin. Goodness-of-fit tests for Copulas: a review and a power study [J]. Insurance: Mathematics and Economics, 2009, 44: 199- 213.

3. Ding Jing, Song Dedun, Yang Ronfu. Further research on application of probability weighted moments in estimating parameters of the Pearson type three distribution [J]. Journal of Hydrology, 1989, 110: 239- 257.

4. Favre, A.-C., Adlouni, S. E., Perreault, L., Thiémonge, N. 
and Bobée, B. Multivariate hydrological frequency analysis using copulas [J]. Water Resources Research, 2004, 40. W01101, doi:10.1029/2003WR002456.

5. Genest, C., Mackay, L.. The joy of copulas: bivariate distributions with uniform marginals. The American Statistician, 1986, 40 (4): 280- 283.

6. Guo Shenglian, Yan Baowei, Xiao Yi, et al. Multivariate hydrological analysis and estimation [J]. Journal of China Hydrology, 2008, 28(3): 1- 7.

7. Jin GY. Similarities and differences of hydrologic frequency distribution models and their parameter estimation problems. Advances in Water Science. 2010, 21(4): 466-470.

8. Michele, C. D. and Salvadori, G.. A generalized pareto intensity-duration model of storm rainfall exploiting 2copulas [J]. Journal of Geophysical Research, 2003, 108(0), XXXX, doi: 10.1029/2002JD002523.

9. Michele, C. D., Salvadori, G., Canossi, M., Petaccia, A. and Rosso, R.. Bivariate statistical approach to check adequacy of dam spillway [J]. ASCE Journal of Hydrological Engineering, 2004, HE/03/22620.

10. Nelsen, R.B.. An introduction to Copulas [J]. SpringerVerlag, New York, 1999.

11. Xu Changjiang, Fan Kexu, Xiao Tianguo. Runoff characteristics and variation tendency of Jinsha River Basin [J]. Journal of Yangtze River, 2011, 41(7): 11- 14, 51.

12. Zhao Wenhuan, Gao Yuan. Analysis on annual and decadal runoff variation characteristics of Jinsha River Basin [J].
Journal of Yangtze River, 2011, 42(6): 98- 100.

13. Sklar, A.. Fonctions de Repartition a' n Dimensions et Leurs Marges [J]. Publishing Institute of Statistical University of Paris 8, 1959: 229- 231.

14. Wang Zhanhai, Chen Yuanfang. Huang Qin, et al. Application study on M-Copula function in flood encounter[J]. Water resources and power, 2009, 27(1): 6973.

15. Xiao Yi. Multivariate Hydrological Analysis and Estimation Methods Based on Copula Function [D]. PhD thesis, Wuhan university, China, 2007.

16. Yang Xiaoyu. Analysis of Runoff Characteristics in Water Transfer Region of the Western Route of the South-toNorth Water Transfer Scheme[D]. PhD thesis, Tianjing university, China, 2008.

17. Ye SZ, Zhan DJ. Engineering Hydrology [M]. 2003, Beijing, China WaterPower Press.

18. Yevjevich, V.. Probability and Statistics in Hydrology [J]. Water Resources Publications, Fort Collins, Colorado, United States, 1972.

19. Zhang L, Vijay P. Singh. Bivariate rainfall frequency distributions using Archimedean copulas [J]. Journal of Hydrology, 2007, 332: 93- 109.

20. Zhang L. Multivariate Hydrological Frequency Analysis and Risk Mapping [D]. PhD thesis, Department of Agricultural and Mechanical College, Louisiana State University, USA, 2005. 